\title{
A Case of Gastric Volvulus in the Emergency Department
}

\author{
Elam A*, Downs A, Burgess M, Dang R, Williams T and Ranasinghe L \\ California North state University College of Medicine, USA
}

*Corresponding author: Annaliese Gabrielle Elam, California North state University College of Medicine (CNUCOM), California, USA

To Cite This Article: Elam A*, Downs A, Burgess M, Dang R, Williams T, Ranasinghe L. A Case of Gastric Volvulus in the Emergency Department. Am J Biomed Sci \& Res. 2021 - 14(2). AJBSR.MS.ID.001970. DOI: 10.34297/AJBSR.2021.14.001970.

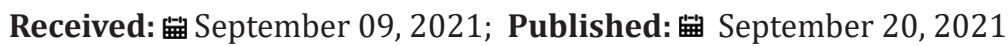

\begin{abstract}
Gastric Volvulus (GV) is a rare condition characterized by rotation of the stomach $180^{\circ}$ around its axis [1]. GV can be either acute or chronic and can lead to various complications including GI obstruction and strangulation. Approximately one third of patients present with acute symptoms [2]. Acute GV is considered a surgical emergency and, if left untreated, has a high rate of mortality. Acute GV is classically characterized by Borchardt's Triad which consists of unproductive retching, epigastric pain, and inability to pass a Nasogastric (NG) tube [3]. Postprandial vomiting can also be seen. Diagnoses are commonly made with imaging (plain radiograph or CT). In adults, GV is commonly due to diaphragmatic defects. We present a patient with a case of acute gastric volvulus in a 74-year-old female with history of hiatal hernia. The patient was diagnosed via imaging and subsequently underwent surgical reduction of the volvulus and hernia repair.
\end{abstract}

Keywords: Gastric Volvulus; Adults; Surgical; Nasogastric; High Rate; Vomiting; Children; Mesenteroaxial; Organ Axial; Diaphragmatic; Anatomical Disorders

\section{Introduction}

Gastric volvulus was first described in an autopsy report from 1866 and first treated in 1897 [3]. The term volvulus derives from the Latin volvere, to turn or roll [4]. Volvulus is characterized as a rotation of the stomach greater than $180^{\circ}$ [5], and symptoms vary based on degree of rotation and speed of onset. Borchardt's classic triad of symptoms is seen in up to $70 \%$ of cases [1]. Single symptoms of dysphagia, epigastric pain and chest pain can occur in $29 \%$ of the cases [2]. Chronic volvulus can present with postprandial pain, vomiting, belching, bloating and satiety [6]. The condition can be classified based on the direction of stomach rotation. The volvulus classifications include type 1 Organ Axial (OA), rotation around an axis from the gastroesophageal junction to the pylorus, or type 2 Mesenteroaxial (MA), rotation around an axis bisecting the lesser and greater curvatures. There are additionally categories including type 3 (combined OA and MA) or type 4(unclassified). OA volvulus is slightly more common, occurring in $59 \%$ of cases [3]. MA volvulus is seen predominantly in children, though it has been noted in adults less commonly [4].
Volvulus can additionally be classified based on etiology as idiopathic/primary or secondary. Primary volvulus is more common and can be associated with structural/anatomical disorders. Secondary volvulus can involve tumors, adhesions, or ligamentous attachments of the stomach. $80 \%$ of cases of GV occur in adults, with the most common underlying cause being diaphragmatic defects such as hiatal or post-traumatic hernias. In children, the condition is often due to congenital diaphragmatic defects. The condition is seen equally in males and females [3]. The case being reviewed is a secondary mesenteroaxial volvulus in an elderly patient with a history of hiatal hernia. Current mortality figures indicate there is a $15-20 \%$ mortality rate from acute gastric volvulus. There have been nonoperative rates as high as $80 \%$ reported in the past [5]. Complications can include perforation, peritonitis, shock, and death [6]. Due to the vague clinical symptoms and rare occurrence of the condition, it is thought to be underdiagnosed. 


\section{Case Presentation}

The patient is a 74-year-old female with a past medical history significant for hypertension, hyperlipidemia, diabetes, Gastroesophageal Reflux Disease (GERD), osteoarthritis, hiatal hernia, and multiple prior abdominal surgeries. Her surgical history includes an appendectomy, 3 C-sections, and a total hysterectomy. She reported that the hiatal hernia has been present for approximately 5 years. She presented to the ED with a chief complaint of left upper quadrant pain and nausea that began one hour after eating lunch that day. She reported moderate, pressurelike, abdominal pain radiating to her left shoulder, rated 5 out of 10 in severity. There was no associated vomiting, diarrhea, or constipation. The patient reported a history of similar postprandial pressure-like abdominal pain and nausea with additional brown emesis 1 week prior, for which she was seen in a different ED and was admitted overnight for NG tube placement and decompression. Patient stated that prior to her previous admission she experienced an insidious onset of shortness of breath. Upon arrival to ED, patient's vital signs were BP: 164/80 HR: 93 RR: 16. She was noted to be in no acute distress despite reporting ongoing abdominal pain.

Exam was significant only for mild epigastric and left upper quadrant abdominal tenderness, with a soft, non-distended abdomen and normal bowel sounds. The patient's vital signs remained stable throughout her ED stay. She was first given IV fluids, pantoprazole, and ondansetron for symptom control during the ED workup. The patient reported significant relief of pain after NG tube decompression. Pain control was continued with IV acetaminophen and morphine. Labs, including CBC with differential, CMP, lactic acid, and PT/PTT were significant for hypokalemia (3.2mmol/L) and mild hyperglycemia $(108 \mathrm{mg} / \mathrm{dL})$. Other lab findings were within normal limits. An abdominal/pelvic CT with contrast revealed a diaphragmatic hernia with displacement of the gastric antrum and pylorus above the level of the GE junction and fundus extending into the left hemithorax through a diaphragmatic defect in a configuration compatible with a mesenteroaxial gastric volvulus and a probable associated gastric outlet obstruction. There was no bowel wall thickening or free perforation noted on CT. The case was then referred to a thoracic surgeon. The patient was transferred to a tertiary care center for surgical repair. The patient underwent an esophagogastroduodenoscopy, a laparoscopic paraoesophageal hernia repair with mesh, and Nissen fundoplication.

Surgical findings included gastric erosion at the gastroesophageal junction and a large hiatal hernia with most of the stomach in the chest cavity. The stomach and mediastinal tissue planes were relatively edematous. The stomach was reduced from the thoracic cavity as much as possible. A laparoscopic Nissen fundoplication was performed to hold the stomach in the correct orientation within the abdominal cavity. There were no complications during the procedure. She was given prophylactic heparin and perioperative IV cefazolin. The patient recovered quickly from surgery. The post-operative chest x-ray and swallow study were normal. The patient did present to the ED several days postoperatively with pleuritic right mid back pain post coughing, but workup was negative. At that time, the abdominal surgical incisions were noted to be well healing and the patient reported normal bowel movements on a full-liquid diet. There were no other complications [Figure 1-3].

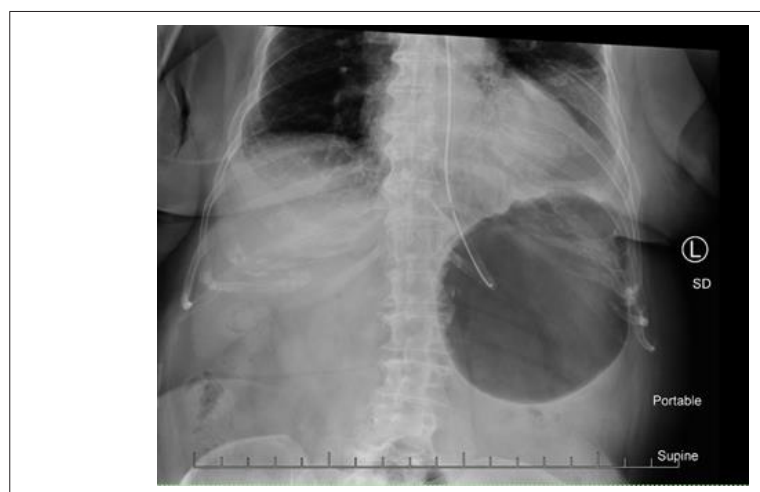

Figure 1: Plain film of the thorax showing the gastric bubble above the diaphragm.

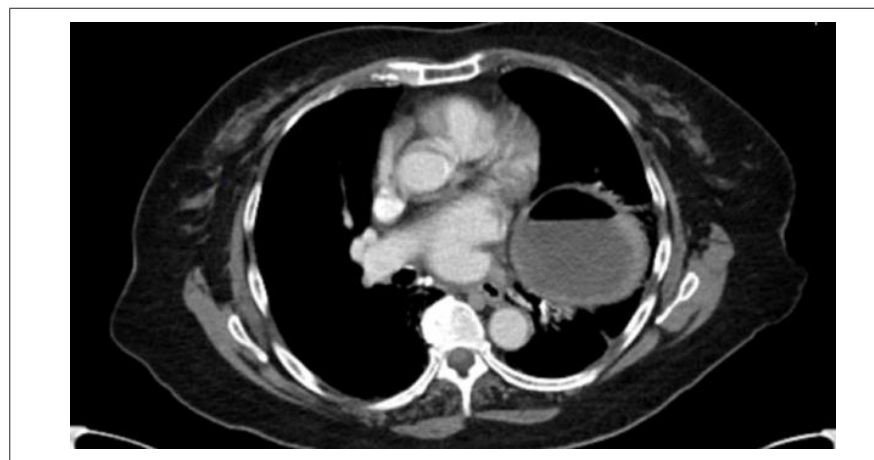

Figure 2: A CT abdomen/pelvis showing displacement of the stomach, indicated by air fluid level, into the left hemithorax, adjacent to the heart.

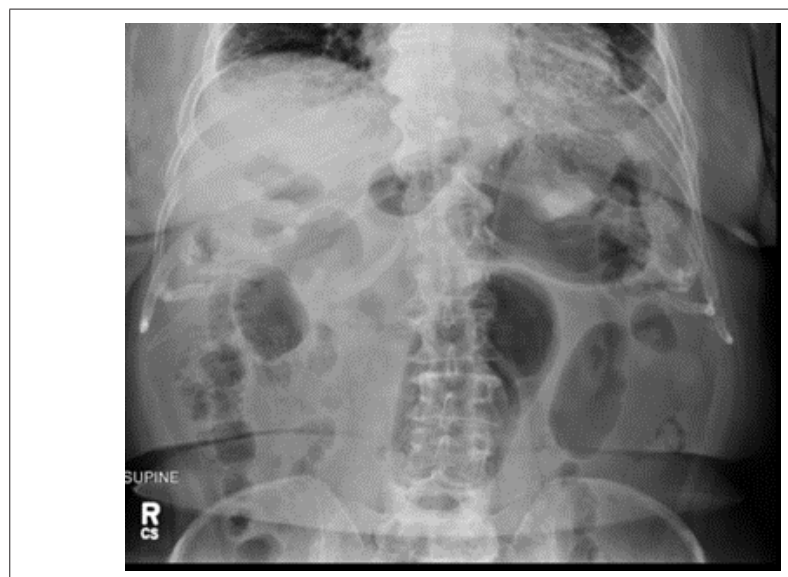

Figure 3: Plain film of the abdomen taken during patient's ED visit several days post-op. 


\section{Differential Diagnoses}

A gastric volvulus is difficult to diagnose due to its nonspecific abdominal symptoms that may resemble many other more common GI etiologies. A gastric volvulus is a diagnosis of exclusion and generally cannot be confirmed without imaging or surgery [4]. Differential diagnoses to consider include hiatal hernia, biliary colic, cholelithiasis, cholecystitis, peptic ulcer disease, gastroesophageal reflux, gastritis, hepatitis, pancreatitis, and acute coronary syndrome.

\section{Imaging}

The workup for patients exhibiting abdominal/chest pain often involves imaging. Since imaging is the main diagnostic tool of gastric volvulus, recent advances in this field have decreased mortality by $15-20 \%$ in volvulus patients [7]. The results of the CT in this case confirmed a typical mesenteroaxial volvulus, which made for a timely diagnosis, and was key to a positive patient outcome. CT is the most used imaging method for diagnosis along with CXR. While barium studies have previously been used as the "gold standard" for diagnosis of GV, CT is now regarded as the superior method. Benefits of CT scans include 24-h access, speed, precise definition of the anatomical defect (allowing operative planning), and assessment of gastric viability and/or perforation (aiding decision regarding urgency of operative intervention). Additionally, a CT does not require an on-site radiologist [8]. CT imaging can also help detect predisposing factors to a gastric volvulus such as diaphragmatic/hiatal hernias, as were seen in this case [3].

The most sensitive and specific diagnostic findings via radiology have been found to be the presence of both an antropyloric transition point without any abnormality at the transition zone and an abnormally located antrum at the same level or higher than the fundus [9]. Additional CT signs of gastric volvulus can include a transition point at the pylorus, a herniation of the antrum into the left hemithorax, an antropyloric junction above the gastroesophageal junction, a reversed position of the greater and lesser gastric curvatures, and stenosis of the gastric segments through the stretched oesophageal hiatus, clearly seen on the coronal reconstruction [9]. Contrast CT images can also show the direction and level of the volvulus, and the location of the transition point (the level of the volvulus) [10]. With a mesenteroaxial volvulus, as seen in this patient, CT will also show antral and distal body herniation into the left hemithorax with an inferiorly located esophagogastric junction below the diaphragm [11].

Initial radiography usually reveals a herniated stomach above the diaphragm, with air fluid levels seen inside the stomach. A chest X-ray may show a retrocardiac gas-filled viscus that can help confirm the diagnosis. Abdominal imaging may reveal a distended viscus in the upper abdomen. An organ axial volvulus may show a horizontal orientation of the stomach with a single air-fluid level and a paucity of distal gas [3]. A mesenteroaxial volvulus may show plain abdominal plain radiographic findings showing a spherical stomach on supine images with two air-fluid levels on erect images with the antrum positioned superior to the fundus [3]. Endoscopy is also used in the evaluation and treatment of a patient with gastric volvulus. Endoscopies are sometimes used to evaluate upper abdominal issues and as a result can reveal signs of gastric volvulus as a byproduct [12].

Endoscopies show a low sensitivity of $32-45 \%[8,13]$ but can sometimes be a helpful follow up in the case of a negative barium swallow [12]. A difficult intubation of the stomach or pylorus can also be highly suggestive of gastric volvulus [3]. Ultrasound may be used as a secondary imaging modality but does not directly show the abnormal position of the bowel. It is helpful in identifying the abnormal positional relationship between the superior mesenteric vein and artery in the case of midgut or colonic volvulus [10]. The vein may be located to the left of the artery, which is the opposite of its usual orientation and suggestive of an abnormality such as gastric volvulus [10]. While not first line, case reports have shown the success of ultrasound as a tool for screening and/or diagnosis of gastric volvulus in both neonates and adults and may be useful in settings without other imaging modalities [14].

\section{Management}

Due to the variety of underlying causes and presentations of this condition, each treatment is established on a case-by-case basis to align with the given patient's state. Generally, primary management of gastric volvulus involves nasogastric tube placement for decompression of the stomach. Immediate surgical consult should follow to prevent gastric ischemia. Most cases will require operative intervention. Emergent laparotomy is performed most. Repair procedures can include hernia repair, gastropexy, gastrectomy, among others. More conservative techniques include laparoscopic surgical repair or percutaneous gastrostomy tube. Few reports compare open versus laparoscopic surgery, though laparoscopic surgery is associated with shorter hospital stays and fewer complications and is preferred in elderly patients and chronic volvulus [1].

\section{Conclusion}

This case provides a useful look at a standard presentation and treatment of a rare and potentially life-threatening condition. Our patient's risk factors and presenting complaints were typical of acute gastric volvulus: an elderly female with a past medical history of chronic hiatal hernia presenting with acute abdominal pain radiating to the scapular region and with a recent history of 
an episode of similar pain one week prior. Though the presentation and risk factors were consistent with an acute gastric volvulus, it is still considered a less likely differential diagnosis due to its low incidence. In this case, as well as in most cases of GV, prompt radiologic studies were key in identifying and confirming the diagnosis. The patient was able to be transferred to a tertiary care center for surgical intervention. Though there is no single "typical" treatment of gastric volvulus established because of the variety of underlying causes and clinical courses experienced by the patients [15], the standard of care includes endoscopy followed by decompression of the stomach. Endoscopy is considered the first step in treating volvulus cases, as it is used to determine what the appropriate next steps are for management [15].

Most commonly, the next step is decompression of the stomach, to draw the stomach back into the abdominal cavity. This is often successful [16]. The patient in the given case received both treatments, and the stomach was reduced back into the abdominal cavity. This was followed by the closure of the hiatal hernia to prevent volvulus recurrence. The patient received a laparoscopic paraoesophageal hernia repair during the same surgery as the endoscopy. This is a minimally invasive repair technique that is considered the standard of care, especially in elderly patients [17]. The patient also underwent a Nissen fundoplication which helps to anchor the stomach into place and to reduce recurrence of herniation in patients with large hiatal hernias [18]. The patient responded well to all first line procedures indicated for volvulus management. The patient recovered well post-surgery with little complications. Several days after the surgery, pleuritic pain was experienced post cough. A workup of the patient was negative for any significant findings.

This pain was most likely due to strain on the surgical repair sites induced by the cough. The patient did not report persistence of pain and pain subsided with the cough. A gastric volvulus is rare and difficult to diagnose. Though it is uncommon it is an important differential diagnosis to consider, as the mortality rate is high if left untreated. Incidence is highest in adults over the age of 50, though the exact incidence is unknown. One of the important risk factors in secondary gastric volvulus is the presence of a hiatal hernia, or other related diaphragmatic abnormalities. Some of the diagnostic challenges are nonspecific symptoms and physical exam findings (vomiting, abdominal pain/tenderness), as well as subtle radiologic findings associated with gastric volvulus. Prompt imaging is crucial in establishing a diagnosis, which allows for timely surgical repair and avoids dangerous complications such as ischemia and perforation [19-25].

\section{References}

1. Rashid F, Thangarajah T, Mulvey D, Larvin M, Iftikhar S (2010) A review article on gastric volvulus: A challenge to diagnosis and management. Inter J Surgery 8(1): 18-24.

2. Cozart JC, Clouse RE (1998) Digestive Diseases and Sciences 43(5): 1057-1060.

3. William W Hope MD, Mohamed Akoad MD, (2018) Gastric Volvulus.

4. Akhtar A, Siddiqui FS, Sheikh AA, Sheikh AB, Perisetti A (2018) Gastric Volvulus: A Rare Entity Case Report and Literature Review. Cureus 10(3): e2312.

5. Costa MR, Matos AS, Almeida JR, Oliveira FJ (2018) Primary gastric volvulus: A report of two cases. J Surg Case Rep 2018(8): 227.

6. Shivanand G, Seema S, Srivastava DN, Pande GK, Sahni P, et al. (2002) Gastric volvulus Acute and chronic presentation. Clin Imaging 27(4): 265-268.

7. Jiro Omata, Katsuyuki Utsunomiya, Yoshiki Kajiwara, Risa Takahata, Nobuo Miyasaka, et al. (2019) Acute Gastric Volvulus Associated with Wandering Spleen in An Adult Treated Laparoscopically After Endoscopic Reduction: A Case Report. Surg Case Rep 2: 47.

8. Light D, Links D, Griffin M (2015) The threatened stomach: management of the acute gastric volvulus. Surgical Endoscopy 30(5): 1847-1852.

9. Millet I, Orliac C, Alili C, Guillon F, Taourel P (2014) Computed tomography findings of acute gastric volvulus. Eur Radiol 24(12): 3115-3122.

10. Peterson, Christine M, et al. (2009) Volvulus of the Gastrointestinal Tract: Appearances at Multimodality Imaging. Radio Graphics 29(5): 1281-1293.

11. Singham S, B Sounness (2009) Mesenteroaxial Volvulus in An Adult: Time Is of The Essence In Acute Presentation. Biomedical Imaging and Intervention J 5(3): 18.

12. Colin Yi-Loong W, Alexander Yaw-Fui C, Albert Su-Chong L, Wai-Keong W (2008) Delayed Diagnosis of Intermittent Mesenteroaxial Volvulus of the Stomach by Computed Tomography: A Case Report. J med case reports 2(1): 343 .

13. Yao-Chun Hsu, Chin-Lin Perng, Chun-Ku Chen, Jai-Jen Tsai, Hwai-Jeng Lin (2010) Conservative Management of Chronic Gastric Volvulus: 44 Cases over 5 Years. World j gastroenterol 16(33): 4200-4205.

14. Y Matsuzaki, M Asai, T Okura, R Tamura (2001) Ultrasonography of Gastric Volvulus "Peanut Sign”. Inter Med 40(1): 23-27.

15.Zuiki Toru, Yoshinori Hosoya, Alan Kawarai Lefor, Hiroyuki Tanaka, Toshihide Komatsubara, et al. (2016) The Management of Gastric Volvulus in Elderly Patients. Int J Surg Case Rep 29: 88-93.

16. Tsang Tat-Kin, Robert Walker, David J Yu (1995) Endoscopic Reduction of Gastric Volvulus: The Alpha-Loop Maneuver. Gastrointest Endosc 42(3): 244-248

17. Rattner, David, Douglas Cassidy, (2016) Laparoscopic Paraesophageal Hernia Repair. J Med Insight.

18. Morino M, Giaccone C, Pellegrino L, Rebecchi F, (2006) Laparoscopic Management of Giant Hiatal Hernia: Factors Influencing Long-Term Outcome. Surg Endos 20(7): 1011-1016.

19. Chaari A, Bahr ME, Khashaba SA, Ismail M, Mahmoud T, et al. (2016) Acute Gastric Volvulus in the Elderly: A Case Report and Review of the Literature. Inter J Gerontol 10(3): 183-185. 
20. Chau B, Dufel S (2007) Gastric volvulus. Emerg Med J 24(6): 446-447.

21. Imperatore K, Olivieri B, Vincentelli C (2016) Acute gastric volvulus: A deadly but commonly forgotten complication of hiatal hernia. Autops Case Rep 6(1): 21-26.

22. Wee J0, Gastric Volvulus in Adults.

23. Teague WJ, Ackroyd R, Watson D I, Devitt PG (2000) Changing patterns in the management of gastric volvulus over 14 years. Br J Surg 87(3): 358-361.
24. Jacob, Carlos Eduardo, Lopasso, Fábio Pinatel, Zilberstein, et al. (2009) Gastric volvulus: A review of 38 cases. ABCD. Brazilian Archives of Digestive Surgery (São Paulo) 22(2): 96-100.

25. Nunes G, Patita M, Fernandes V, Fonseca J, (2017) Paraesophageal hernia and gastric volvulus: an uncommon etiology of vomiting and upper gastrointestinal bleeding. Rev Esp Enferm Dig 109(4): 294-295. 